\title{
The Training and Performance of Emergency Physicians as Anesthetists for International Medical Surgical Response Teams: The Emergency Physician's General Anesthesia Syllabus (EP GAS)
}

\author{
Richard Skupski $^{1,2,3}$, Mark Walsh ${ }^{1,2,3 *}$, Manar Jbara ${ }^{1}$, Donald Zimmer ${ }^{1,2,4}$, Bhavesh M. Patel ${ }^{2,5}$, \\ Michael T. McCurdy ${ }^{2,6}$, James Lantry ${ }^{2,6}$, Braxton Fritz ${ }^{1}$, Patrick Davis ${ }^{1,3}$, Harsha Musunuru ${ }^{1}$, \\ Anne Newbold ${ }^{1}$, Art Toth ${ }^{1}$, Richard Frechette ${ }^{2}$, Tiffany Alexander ${ }^{1}$, Madhura Sundararajan', \\ John Lovejoy ${ }^{3}$, Dan Hottinger ${ }^{1,2}$, Joe Capannari ${ }^{1}$, Rachel Kurcz ${ }^{1}$, Gerard Bernard ${ }^{3}$, Harold Previl ${ }^{3}$ \\ ${ }^{1}$ Department of Anesthesia and Trauma Services, Memorial Hospital of South Bend, South Bend, USA; ${ }^{2}$ St. Damien and St. Luc's \\ Hospital, Port au Prince, Haiti; ${ }^{3}$ Sacred Heart Hospital, Milot, Haiti; ${ }^{4}$ Mayo Clinic_Emergency Medicine Residency Program, \\ Rochester, USA; ${ }^{5}$ Mayo Clinic_-Critical Care Medicine, Scottsdale, USA; ${ }^{6}$ University of Maryland School of Medicine, Baltimore, \\ USA. \\ Email: ${ }^{*}$ markwalshmd@gmail.com
}

Received November $25^{\text {th }}$, 2013; revised January $12^{\text {th }}, 2014$; accepted January $18^{\text {th }}, 2014$

Copyright (C) 2014 Richard Skupski et al. This is an open access article distributed under the Creative Commons Attribution License, which permits unrestricted use, distribution, and reproduction in any medium, provided the original work is properly cited. In accordance of the Creative Commons Attribution License all Copyrights (C) 2014 are reserved for SCIRP and the owner of the intellectual property Richard Skupski et al. All Copyright (c) 2014 are guarded by law and by SCIRP as a guardian.

\section{ABSTRACT}

Background: The availability of an anesthesiologist is often a limiting factor in the number of operations that can be performed by International Medical Surgical Response Teams (IMSuRT). Because emergency physicians (EPs) possess skills in airway control, management of moderate and deep sedation, and ventilator management, we propose that with proper training in general anesthesia, EPs can serve as anesthetists for IMSuRT with anesthesiologist supervision. Methods: During a 10-week period, a board-certified EP administered general anesthesia to 60 patients prior to a surgical medical mission trip. The breakdown of surgical cases was: 11 orthopedic, 2 genitourinary, 20 ear, nose, and throat, 8 obstetrics and gynecological, 13 general surgery, and 6 vascular. A simplified protocol for induction, maintenance, and emergence was adhered to for all cases. Results: Fourteen orthopedic cases using general anesthesia were performed in a one-week period in Haiti. These cases involved open reduction and internal fixation (ORIF), hemiarthoplasty, hardware removal, tendon transfer and external fixation of fractured bone. Conclusion: We demonstrate the feasibility of a model curriculum to train EPs in the basics of anesthesia. The EP can safely and effectively deliver general anesthesia for major cases on surgical medical mission trips under the auspices of an anesthesiologist in an austere environment.

\section{KEYWORDS}

Emergency Physicians; General Anesthesia Syllabus; International Medical Surgical Response Teams; Anesthetists; Induction; Maintenance; Emergence; Anesthesia

\section{Introduction}

In the developing world, the morbidity and mortality of general anesthesia remain very high [1-3]. Anesthesiologists are not readily available in this environment and their absence limits the performance of surgical proce-

\footnotetext{
"Corresponding author.
}

dures in the developing world and for International Medical Surgical Response Teams (IMSuRT) or disaster relief programs [3-5]. Because emergency physicians (EPs) possess skills of airway management, expertise in moderate and deep sedation, and ventilator management, they, after proper training in general anesthesia, could be capable of serving as anesthetists under the direction of 
anesthesiologists during surgical mission trips in developing countries [6] much as certified registered nurse anesthetists (CRNA) perform in the United States under the close supervision of anesthesiologists.

With a skill set largely paralleling that of an anesthetist, an EP is an ideal provider to assist the anesthesia team. EPs: 1) have airway management skills, including bagvalve mask ventilation and endotracheal intubation; 2) are familiar with commonly used drugs in the operating room, including opioids, benzodiazepines, propofol, paralytics, and ketamine; and 3) frequently provide critical care management with regards to fluid resuscitation, vasopressor use and ventilator management. Therefore, EPs are capable of functioning as anesthetists on surgical mission trips where anesthesiologists are in short supply. This program has been designed to meet the increased demands of the general anesthetic need in developing countries while maintaining transparency, accountability, and safety.

\section{Methods}

We describe training undertaken by EPs that could be implemented for other surgical mission trips and disaster relief programs, wherein the EP functioned as an anesthetist supervised by anesthesiologists during surgical cases. 60 surgical cases were performed at the home institution prior to the medical mission, during which time the EP administered anesthesia under the direct supervision of a board-certified anesthesiologist. Subsequent to this training, the EP performed fourteen general anesthesia cases in a Haitian hospital under the supervision of an anesthesiologist during a one-week period in February 2011 and again in 2013.

In accordance with other international medical and surgical relief publications, medical standard of care was met by continual supervision of the EP by an anesthesiologist from the pre- to post-operative time in the United States and in Haiti [7-9]. Therefore, institutional review board (IRB) approval was waived. No statistical analysis was used in this descriptive study.

\section{Results}

\subsection{Training of the Emergency Physician as an Anesthetist in the US}

The curriculum for this program, known as Emergency Physician's General Anesthetic Syllabus (EP GAS), is centered on Stoelting and Miller's 'The Basics of Anesthesia' textbook [10]. Emphasis was placed on the chapters addressing the anesthesia machine, properties of inhaled anesthetics, mean alveolar concentration of gas principle, second gas effect, onset and relative potencies of narcotics, benzodiazepines, muscle relaxants, local anesthetics, and conduction anesthesia.

Initial training of the board-certified EP started in the United States at Memorial Hospital in South Bend, Indiana, with a basic introduction to the anesthesia machine. The board-certified anesthesiologist supervised the EP in the same fashion that anesthesiologists already supervise the training of paramedics, emergency medicine residents, and certified registered nurse anesthetists as defined by the American Society of Anesthesiologists. In addition, the anesthesiologist provided instruction on managing the difficult airway, per existing curricula $[6,11,12]$.

The EP learned how to set up for a routine anesthesia case. "MS MAID" was the simple, commonly used mnemonic applied:

M-Machine check (on/off switch, oxygen $\left(\mathrm{O}_{2}\right)$, nitrous oxide $\left(\mathrm{N}_{2} \mathrm{O}\right)$, sevoflurane, and desflurane levels, filling apparatus with anesthetic, pop-off valve pressure check, and circuitry maintenance).

S-Suction apparatus assembly.

M-Monitor (Blood pressure (BP), Heart rate (HR), $\mathrm{O}_{2}$ saturations, end tidal $\mathrm{CO}_{2}\left(\mathrm{ETCO}_{2}\right)$, anesthetic inspiratory and expiratory concentrations, ventilator monitor and settings).

A-Airway supplies (endotracheal tube, stylet, laryngoscope blade and handle, bag valve mask, oral airway, $\mathrm{O}_{2}$ tubing, tape).

I-Intravenous line placement.

D-Drugs (Table 1).

During a 10-week period, the EP provided general anesthesia for 60 patients (Table 2). The anesthesiologist visually monitored the measuring and administration of medications at all times. The anesthesiologist was present for the duration of each surgical case, rendering instruction and requiring the EP to perform a regularly repeated survey of the patient, equipment, and therapies. The EP's repeated survey included a visual inspection of the patient, airway device, machine and its circuit integrity, $\mathrm{O}_{2}$ saturation, end-tidal carbon dioxide $\left(\mathrm{ETCO}_{2}\right)$, inhaled and exhaled gas concentration, minute ventilation (MV), tidal volume (TV), respiratory rate, and maximum airway pressure. The EP also performed repeated physical exam assessments, which included palpation of the anterior auricular artery (a branch of superficial temporal artery), assessment of scalp capillary refill, and auscultation of the lungs with a standard and/or esophageal stethoscope. In addition, during cases with spontaneous ventilation and general anesthesia, the tidal volume and minute ventilation were continually monitored. After surgery, both the anesthesiologist and the EP accompanied patients from the operating room to the recovery room where the EP gave report to the postoperative nurse. The anesthesiologist supervised the EP for the entire duration of each case from pre- to post-op. To 
The Training and Performance of Emergency Physicians as Anesthetists for International Medical

Surgical Response Teams: The Emergency Physician’s General Anesthesia Syllabus (EP GAS)

Table 1. Medications used for anesthesia during surgical cases.

\begin{tabular}{ccc}
\hline Steps & Drugs \\
\hline & 1 & Preoxygenation $100 \% \mathrm{O}_{2}$ \\
Pre-Induction \& Induction & 3 & Rocuronium $5 \mathrm{mg} / 0.5 \mathrm{~mL}$ Glycopyrrolate $0.2 \mathrm{mg} / 1 \mathrm{~mL}$ \\
& 4 & Fentanyl $100 \mathrm{mg} / 2 \mathrm{~mL}$ \\
Propofol $150 \mathrm{mg} / 15 \mathrm{~mL}$
\end{tabular}

Table 2. US training cases.

\begin{tabular}{|c|c|c|c|}
\hline Surgical Category & $\begin{array}{l}\text { No. of } \\
\text { Cases }\end{array}$ & $\begin{array}{l}\text { Example } \\
\text { Cases }\end{array}$ & Example Challenges \\
\hline Orthopedic & 11 & Hip prosthesis & Elderly patients who require vasopressor support \\
\hline Genitourinary & 2 & Prostatectomy & Attention to blood and volume loss \\
\hline Ear, nose, throat & 20 & Thyroidectomy & Covered airway with temporary circuit disconnection. Condensation in $\mathrm{ETCO}_{2}$ connector. \\
\hline Obstetrics and gynecology & 8 & $\begin{array}{c}\text { Vaginal } \\
\text { Hysterectomy }\end{array}$ & Required 3 doses of ephedrine and 1 dose of phenylephrine after fluid bolus for hypotension \\
\hline General surgery & 13 & Colectomy & Nasogastric tube enters trachea and deflates bellows \\
\hline Vascular & 6 & Endarterectomy & Inadvertent shutting off of $\mathrm{ETCO}_{2}$ monitor \\
\hline
\end{tabular}

prepare for particularly austere environments where electricity can be suddenly lost, the EP must know how to provide anesthesia without functioning ventilators or instrument panels on the machines. Therefore, training further focused on administering general anesthesia by Bag Ventilation (BV) of intubated patients and Bag Mask Ventilation (BMV) of non-intubated patients. Endotracheal intubation was preferred to laryngeal mask airway (LMA) to avoid problems with LMA dislodgment, dead space ventilation, and intra-operative laryngospasm caused by possible light anesthesia or aspiration. Adequate alveolar ventilation was monitored with lung auscultation and visual inspection of the reservoir bag. Because of the possibility of inconsistent access to pulse oximetry and capnography, training included recognition of hypopneic hypoventilation, which pulse oximetry may not detect $[3,13]$. Further emphasis was placed on mask ventilation skills for induction and emergence, as well as management of post extubation laryngospasm with suc- cinylcholine or propofol rescue.

\subsection{Intraoperative Anesthesia Administration}

Following pre-oxygenation, the EP used a standard intravenous induction regimen that included premedication with glycopyrrolate $0.2 \mathrm{mg}$, rocuronium $5 \mathrm{mg}$, and fentanyl $100 \mu \mathrm{g}$. Induction was then performed with propofol $150 \mathrm{mg}$ and succinylcholine $100 \mathrm{mg}$. Following endotracheal intubation and securing of the endotracheal tube, 10 liters per minute $(\mathrm{L} / \mathrm{min})$ of $\mathrm{O}_{2}$ were administered with either sevoflurane $3 \%$ - $4 \%$ or desflurane $5 \%$ $6 \%$, depending on availability, in order to speed uptake of the anesthetic. The patient was carefully positioned to avoid excess tissue pressure, the eyes were taped shut and then the patient was draped. Prior to incision, $\mathrm{O}_{2}$ and $\mathrm{N}_{2} \mathrm{O}$ flow rates were both set at $2 \mathrm{~L} / \mathrm{min}$. After incision, $\mathrm{O}_{2}$ and $\mathrm{N}_{2} \mathrm{O}$ flows were decreased to $0.6 \mathrm{~L} / \mathrm{min}$ each for the duration of the case. Anesthetic enhancement for light anesthesia was handled with boluses of either pro- 
pofol $50 \mathrm{mg}$ or fentanyl $50-100 \mu \mathrm{g}$. During short cases ( $<45$ minutes), spontaneous ventilation was permitted to return, and in longer cases ( $>45$ minutes), a standard dose of rocuronium $30 \mathrm{mg}$ was given with mechanical ventilation. Standard ventilator settings for an adult male of 70 $\mathrm{kg}$ weight were: tidal volume of $700 \mathrm{~mL}$, respiratory rate of 8 breaths/min, maximum peak pressure of $40 \mathrm{~cm}$ of $\mathrm{H}_{2} \mathrm{O}, \mathrm{FiO}_{2}$ between $40 \%-50 \%$. These ventilator settings were adjusted in accordance with patient weight and gender. Ventilator settings were adjusted to keep $\mathrm{ETCO}_{2}$ between 30 - $40 \mathrm{mmHg}$. In longer cases with paralysis by rocuronium, doses of neostigmine $3 \mathrm{mg}$ and glycopyrrolate $0.4 \mathrm{mg}$ were available, if necessary, for paralysis reversal prior to extubation. As the last suture was applied, sevoflurane, desflurane, and $\mathrm{N}_{2} \mathrm{O}$ were discontinued and washed out by increasing the $\mathrm{O}_{2}$ flow to 10 $\mathrm{L} / \mathrm{min}$. Patient emergence from anesthesia was most often characterized by eye opening and purposeful motor activity, such as reaching for the endotracheal tube. Once awake, the patient was extubated and transported to the postoperative recovery unit where the EP stayed with the patient until airway protection was guaranteed.

\subsection{The Haiti Experience}

From February 19th to 26th, 2011, one year following the earthquake of 2010, the Haitian IMSuRT consisted of five orthopedic surgeons, two anesthesiologists, and one EP serving as an anesthetist and critical care physician. Surgery was performed at Sacred Heart, Milot, Haiti, a private hospital. The mission team leader reviewed the scheduled cases and decided that the EP would serve as an anesthetist for the longer surgical cases in the central operative suite. This enabled the two anesthesiologists to concentrate on emergency, pediatric, and quick-turnover cases, while also attending to any preoperative and postoperative care. The cases for which the EP gave anesthesia are listed below (Table 3).

For the first three cases done by the EP, a board-certified anesthesiologist was present during induction. In these instances, the patient was in a stable maintenance mode before the anesthesiologist entrusted patient care to the EP. As the anesthesiologists were busy with other cases and duties, the EP emerged all fourteen patients in the Operating Room (OR) and subsequently provided postoperative care in the crowded postoperative unit, which also served as a preoperative and trauma unit. Despite causing significant delays in start times for subsequent surgeries, a policy was established to require the EP anesthetist to remain with postoperative patients until their postoperative aspiration risk was negligible. Also, the anesthesiologists and EP rounded nightly on both critically ill patients and all postoperative patients. A
Table 3. Haiti surgical cases.

\begin{tabular}{ccccc}
\hline Case & Age & Sex & DOS & Procedure \\
\hline 1 & 33 & M & $2 / 21 / 11$ & Left hip hemiarthroplasty ${ }^{1}$ \\
2 & 9 & F & $2 / 21 / 11$ & Drilling, incision and drainage \\
3 & 19 & M & $2 / 21 / 11$ & Right closing wedge osteotomy \\
4 & 35 & M & $2 / 21 / 11$ & Removal of ex-fix, long leg cast ${ }^{2}$ \\
5 & 28 & F & $2 / 23 / 11$ & Removal of hardware \\
6 & 56 & F & $2 / 23 / 11$ & Exploration of sternal mass \\
7 & 36 & M & $2 / 23 / 11$ & Removal of hardware distal interlock \\
8 & 29 & M & $2 / 23 / 11$ & Removal of hardware \\
9 & 17 & M & $2 / 24 / 11$ & Open ACL reconstruction \\
10 & 30 & F & $2 / 24 / 11$ & External fixation (long) \\
11 & 26 & M & $2 / 24 / 11$ & Right forearm tendon transfer \\
12 & 50 & F & $2 / 25 / 11$ & ORIF R ankle (Asthma) \\
13 & 27 & M & $2 / 25 / 11$ & I\&D, ex-fix R femur \\
14 & 28 & M & $2 / 25 / 11$ & Extensor R forearm tendon transfer \\
\hline
\end{tabular}

${ }^{1}$ First case, Emergency Physician left alone by anesthesiologist for emergency vascular access in cholera tent. ${ }^{2}$ Failure to give cefazolin preoperatively. ${ }^{3}$ Case aborted due to unexpected heavy bleeding and uncertain anatomic extension of mass. ${ }^{4}$ Delayed emergence due to unexpected prolongation of surgery. DOS = date of surgery. ACL = anterior cruciate ligament.

similar course of care was given during a return trip in February-March 2013.

While in Haiti, the EP had to stabilize the airway of three postoperative patients by jaw thrust, rescue position, and suctioning. Furthermore, because only one portable suction device existed, the EP recruited paramedical personnel to carefully watch at-risk postoperative patients. The challenges encountered by the EP in the US training cases and in Haiti are summarized below (Table 4).

\section{Discussion}

The shortage of anesthesiologists in developing countries severely limits the ability to perform needed surgeries [2-4]. For example, in Rwanda, there are only 14 anesthesiologists in a country of 11 million people [14]. In our previous mission trip, we noted how essential the anesthesiologist was to the success of relief efforts immediately after the Haitian earthquake on January 12, 2010. At that time, Sacred Heart Hospital had 74 hospital beds and two operating rooms. After the earthquake, the hospital increased capacity to 430 beds with six operating rooms. Due to the shortage of both space and anesthesiologists in the post-earthquake period, many debridements and external fixator revisions were done with 
The Training and Performance of Emergency Physicians as Anesthetists for International Medical

Surgical Response Teams: The Emergency Physician’s General Anesthesia Syllabus (EP GAS)

Table 4. US training challenges and challenges in Haiti.

\begin{tabular}{|c|c|c|c|}
\hline & US training challenges & & Haiti challenges \\
\hline 1 & Patients with large body habitus. & 1 & Lack of anesthetic tubing requiring reuse. \\
\hline 2 & Vapor lock with sevoflurane not filling. & 2 & Inconsistent wall suction. \\
\hline 3 & Prone position and pressure point protection. & 3 & Rationing of $\mathrm{O}_{2}, \mathrm{~N}_{2} \mathrm{O}$, sevoflurane tanks. \\
\hline 4 & Laryngospasm treatment. & 4 & Malfunctioning $\mathrm{ETCO}_{2}$ monitor. \\
\hline 5 & Flat reservoir bag due to leaky scavenger valve. & 5 & $\begin{array}{l}\text { Asthma presented on table requiring albuterol } \\
\text { administration in ET tube with sevoflurane. }\end{array}$ \\
\hline 6 & $\begin{array}{l}\text { Nasogastric tube placement entering the trachea } \\
\text { which deflates bellows on ventilator. }\end{array}$ & 6 & Rudimentary C-arm and orthopedic tourniquets. \\
\hline 7 & Beta-blockers for tachycardia. & 7 & Refilling of Sodalime. \\
\hline 8 & Diagnosis of dead space ventilation. & 8 & Zeroing of $\mathrm{ETCO}_{2}$ and $\mathrm{O}_{2}$ causes 5 seconds without monitoring. \\
\hline \multirow[t]{5}{*}{9} & $\begin{array}{l}\text { Delayed post induction oral airway insertion to } \\
\text { prevent biting down on tube during emergence. }\end{array}$ & 9 & $\begin{array}{l}\text { Preparation of drugs and equipment for } \\
\text { unanticipated arrival of cases. }\end{array}$ \\
\hline & & 10 & Lack of syringe labels. \\
\hline & & 11 & Shortage of blunt needles. \\
\hline & & 12 & Shortage of IV extension tubing. \\
\hline & & 13 & Setting-up delays due to OR changes and unfamiliarity with rooms. \\
\hline
\end{tabular}

midazolam, ketamine, and regional anesthesia in tents under the supervision of the EP. Many additional cases, including more complicated cases, could have been managed by general anesthesia if nurse anesthetists or anesthesiologists had been available [15]. Our experience demonstrates how EPs can fill this void.

Morbidity and mortality of general anesthesia are very high in the developing world [1-3,10]. The Millennium Development Goals, an assessment generated by the United Nations, found that the incidence of anesthesia accidents is greater in the developing world due to the lack of modern anesthetics and monitoring devices [16-19]. The World Health Organization's “Safe Surgery Saves Lives” initiative has identified safe anesthesia as a public health priority in low- and middle-income countries. Mortality associated with surgery, which is typically between $0.4 \%-0.8 \%$ in industrialized countries, is up to 10 times higher in developing countries [20]. The shortage of needed surgical facilities capable of providing general anesthesia has resulted in increased use of local and spinal anesthesia as well as the use of ketamine [19]. Additionally, reports exist of nurse-administered ketamine for general anesthesia without physician supervision [9]. Due to increased interest in disaster relief efforts, a defined standard of care has been proposed for general anesthesia in the developing world. In addition, transparency and accountability are essential for EPs delivering general anesthesia for IMSuRT $[7,8]$.

In the austere medical environment where the shortage of anesthesiologists often remains the rate-limiting step for surgery, the EP skilled in airway and ventilator man- agement and trained in the basics of operative anesthesia is prepared to deliver general anesthesia under the supervision of an anesthesiologist. Quality assurance, transparency, and accountability for EPs delivering general anesthesia in the austere medical environment are essential components of the EP GAS curriculum $[7,8]$.

The present training regimen was designed specifically to provide necessary anesthesia while avoiding the complications characteristic of developing world general anesthesia, namely technical failure and human error. Technical errors include equipment design, improper drug dosage, disconnection of or inadequate circuitry, inadequate gas flows, and esophageal intubation [19,21, 22]. Human error is subdivided into four categories: 1) skill deficiency; 2) poor judgment; 3) lack of sufficient monitoring; and 4) inattention. Prevention of anesthetic mishaps begins in the preoperative phase and requires constant intra-operative vigilance [23]. We have attempted to address human and technical errors that cause anesthetic mishaps by developing a curriculum specifically designed for the EP. We describe a very specific protocol that can be adapted to many situations requiring general anesthesia in austere environments. Training with strict adherence to the set protocol during the 60 initial supervised cases prepared the EP to single-handedly administer anesthesia to 14 orthopedic cases in an austere environment. Constant vigilance to the developed checklists and cues ensured safe anesthesia. For instance, adhering to the initial MS MAID survey and repeating the intraoperative survey checklists allowed for reliable safety during induction, maintenance, and emergence 
from general anesthesia (Table 5). The specific components of the checklist are reiterated by the mnemonic MS MAID: pre-induction checking of the anesthetic Machine, Suction, Monitors, Airway supplies, IV site confirmation, and proper Drug labeling of the syringes in a repetitive fashion. For induction, two small syringes, one large syringe, and one medium syringe were used as visual cues to prevent syringe swap errors. These visual cues decreased human error by helping cue the EP to draw up and administer the correct amount of medication throughout the case. In addition to the four syringes, the EP also had propofol and fentanyl ready for supplemental dosing for longer cases (Figure 1). Checklists like this have been shown to reduce medical errors, including

Table 5. Initiation, maintenance and emergence from Anesthesia.

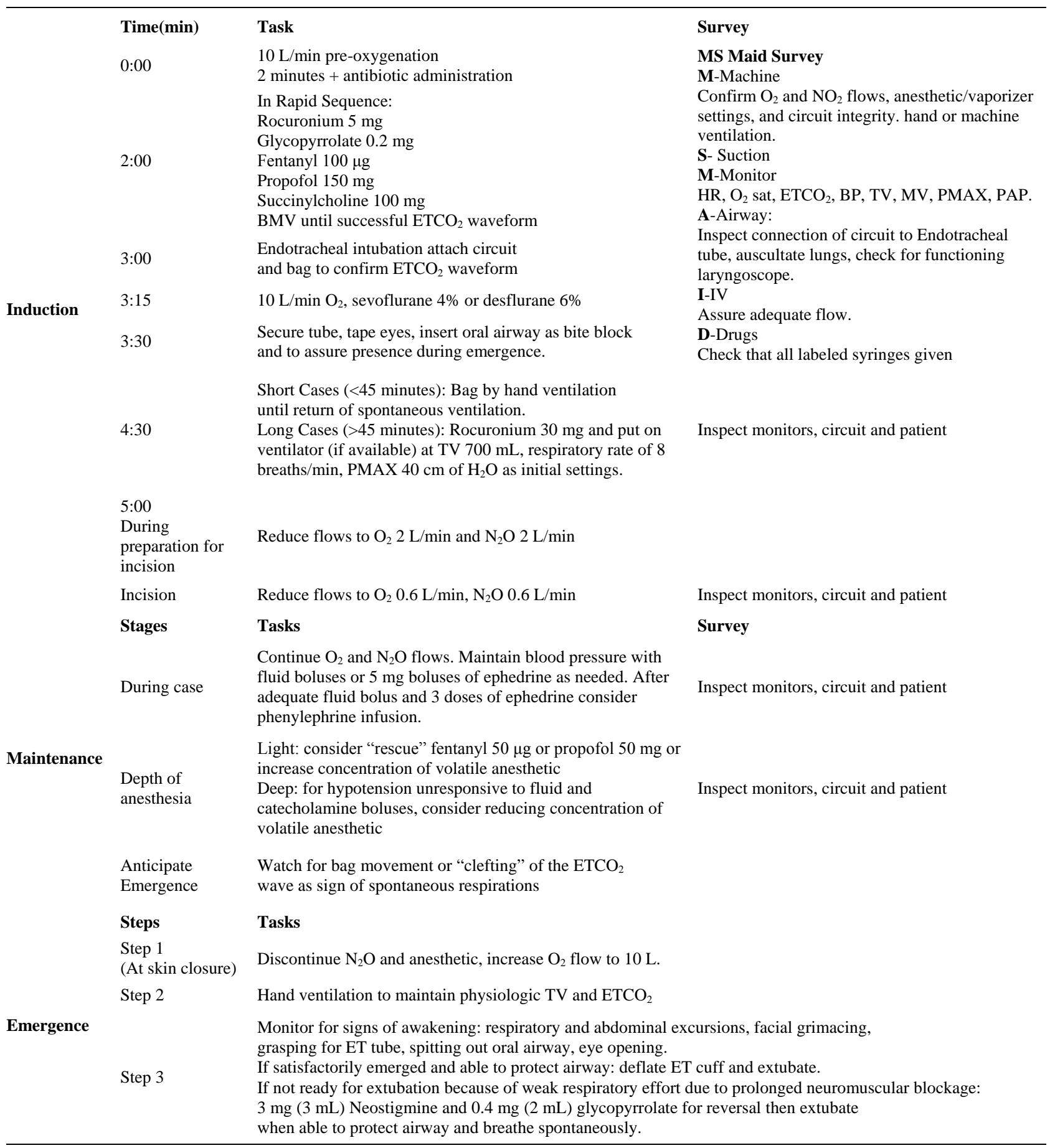




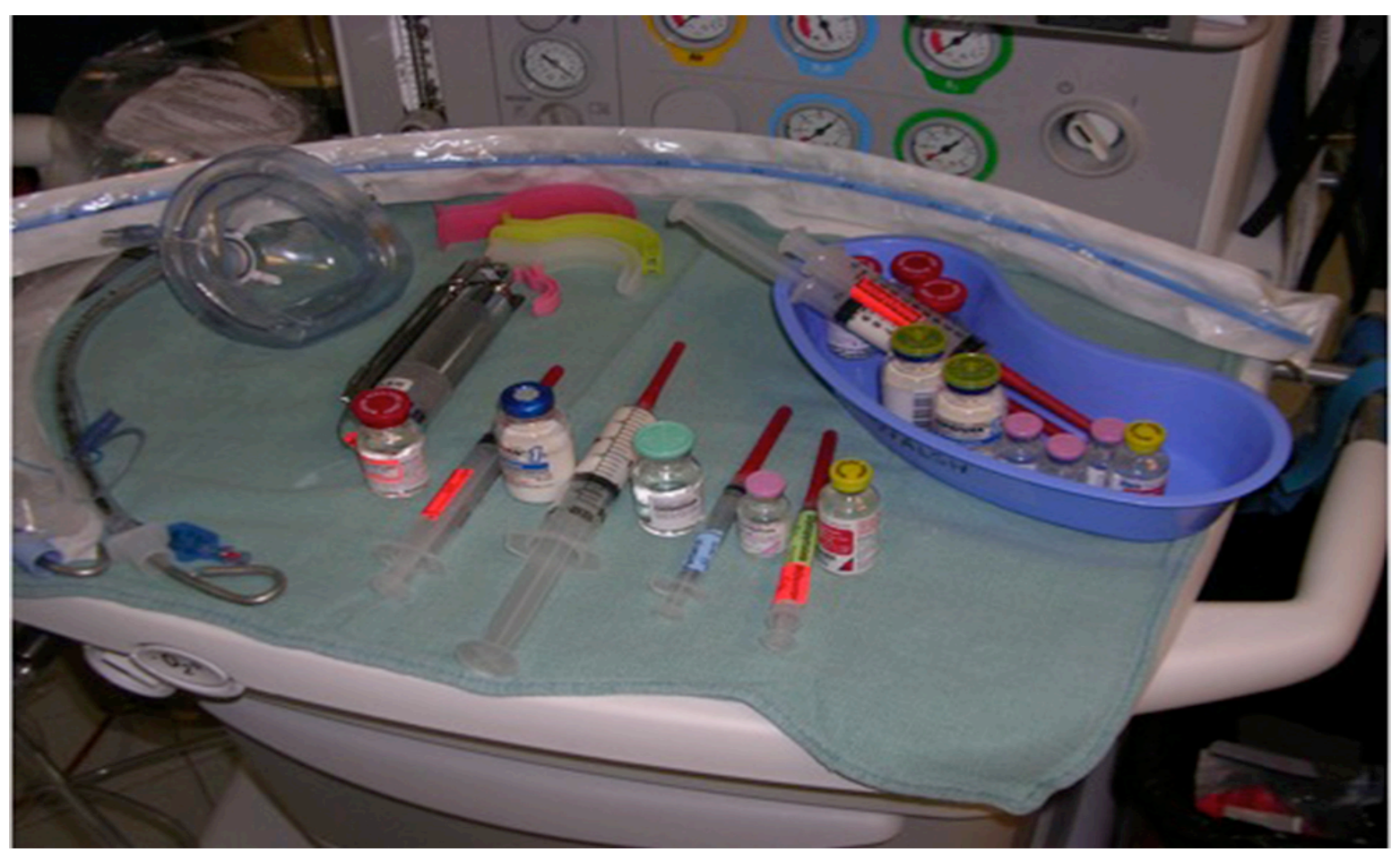

Figure 1. Set up labeled syringes (“two small, one large and one medium” for induction) with "rescue” propofol, succinylcholine, and medications for the next case in basin. Endotracheal tube with blade, stylet, oral airways, and Bougie with mask ready. Drugs used during the surgical cases: One small $3 \mathrm{~mL}$ syringe of rocuronium $5 \mathrm{mg}(0.5 \mathrm{~mL}) /$ glycopyrrolate $0.2 \mathrm{mg}(1$ $\mathrm{mL}$ ), one small $3 \mathrm{~mL}$ syringe of fentanyl $100 \mu \mathrm{g}(2 \mathrm{~mL})$, one large $20 \mathrm{~mL}$ syringe with $150 \mathrm{mg}$ propofol (15 mL), one medium $10 \mathrm{~mL}$ syringe succinylcholine $100 \mathrm{mg}(5 \mathrm{~mL})$, one small $3 \mathrm{~mL}$ syringe of rocuronium $30 \mathrm{mg}(3 \mathrm{~mL})$ for cases lasting more than 45 minutes (not shown), two $10 \mathrm{~mL}$ syringes contain rescue doses of propofol $50 \mathrm{mg}(5 \mathrm{~mL})$ and succinylcholine $100 \mathrm{mg}$ (5 mL).

in the operating room [24-26].

The EP GAS we developed is not the first time physicians have trained to serve as anesthesiologists with limited and focused instruction and caseload. During World War II, the University of Wisconsin began offering an accelerated 90-day medical schooling and specialization for anesthesia. The legendary anesthesiologist Virgil Stoelting was one of the first 17 graduates.

This was done in order to meet the increasing demand for anesthesiologists [27]. Today, the shortage of anesthesiologists in both the developed and developing world, limits the ability to provide safe and effective anesthesia $[28,29]$, however, the properly trained and supervised modern EP can fill this void. The increased emphasis on emergent airway management by EPs has extended their airway skill set to include the administration of anesthetic agents and muscle relaxants during tracheal intubation [6]. These expanded skills establish the groundwork upon which the EP can build credible experience as an anesthetist in austere surgical environments.

\section{Conclusion}

EPs can effectively fill the void between the need for and the limited supply of anesthesiologists in austere medical environments. No formal curriculum exists to teach a non-anesthesiologist the basics of general anesthesia administration. Our training curriculum that included textbook reading about key anesthesiology principles, 60 anesthesiologist-supervised cases, and frequent use of the MS MAID mnemonic, prepared an EP to serve as an anesthetist in an austere environment (Table 5). We demonstrate the feasibility of a model curriculum for training EPs in the basics of anesthesia. The EP can effectively deliver general anesthesia for major cases on surgical medical mission trips under the auspices of an anesthesiologist.

\section{Acknowledgements}

The authors would like to thank the many Haitian physicians, nurses, and other medical and administrative support staff for their invaluable assistance with this project.

\section{REFERENCES}

[1] M. Dünser, I. Baelani and L. Ganbold, "The Specialty of Anesthesia Outside Western Medicine with Special Con- 
sideration of Personal Experience in the Democratic Republic of the Congo and Mongolia,” Der Anaesthesist, Vol. 55, No. 2, 2006, pp. 118-132.

http://dx.doi.org/10.1007/s00101-006-0979-6

[2] B. T. Ugwu and E. L. Isamade, "Interoperative Cardiac Arrest-A Tropical Experience,” West African Journal of Medicine, Vol. 19, No. 4, 2000, pp. 277-280.

[3] I. A. Walker and I. H. Wilson, "Anaesthesia in Developing Countries-A Risk for Patients.” The Lancet, Vol. 371, No. 9617, 2008, pp.968-969. http://dx.doi.org/10.1016/S0140-6736(08)60432-8

[4] S. Jochberger, F. Ismailova, W. Lederer, V. Mayr, G. Luckner and V. Wenzel, "Anesthesia and Its Allied Disciplines in the Developing World: A Nationwide Survey of the Republic of Zambia,” Anesthesia \& Analgesia, Vol. 106, No. 3, 2008, pp. 942-948.

http://dx.doi.org/10.1213/ane.0b013e318166ecb8

[5] K. W. Grathwohl and S. G. Venticinque, "Organizational Characteristics of the Austere Intensive Care Unit: The Evolution of Military Trauma and Critical Care Medicine; Applications for Civilian Medical Care Systems," Critical Care Medicine, Vol. 36, No. 7, 2008, pp. S275-S283. http://dx.doi.org/10.1097/CCM.0b013e31817da825

[6] C. L. Gwinnutt, "The Interface between Anaesthesia and Emergency Medicine,” Emergency Medicine Journal, Vol. 18, No. 5, 2001, pp. 325-239. http://dx.doi.org/10.1136/emj.18.5.325

[7] C. H. Schultz and G. J. Annas, "Altering the Standard of Care in Disasters-Unnecessary and Dangerous,” Annals of Emergency Medicine, Vol. 59, No. 3, 2012, pp. 191195.

http://dx.doi.org/10.1016/j.annemergmed.2011.07.037

[8] K. Peleg and A. L. Kellerman, "Medical Relief after Earthquakes: It's Time for a New Paradigm," Annals of Emergency Medicine, Vol. 59, No.3, 2012, pp. 188-190. http://dx.doi.org/10.1016/j.annemergmed.2011.07.028

[9] M. Bisanzo, K. Nichols, H. Hammerstedt, B. Dreifuss, S. Nelson, S. Chamberlain, et al., "Nurse-Administered Ketamine Sedation in an Emergency Department in Rural Uganda,” Annals of Emergency Medicine, Vol. 59, No. 4, 2012, pp. 268-275. http://dx.doi.org/10.1016/j.annemergmed.2011.11.004

[10] R. K. Stoelting and R. D. Miller, "Basics of Anesthesia," 5th Edition, Churchil Livingstone, Inc., New York, 2007, pp. 1-697.

[11] A. H. Giesecke and W. H. Montgomery, "The Role of Anesthesiologists in Paramedic Training," American Society of Anesthesiologists Newsletter, Vol. 69, 2005, p. 11.

[12] L. C. Berkow, R. S. Greenberg, K. H. Kan, E. Colantuoni E, et al., "Need for Emergency Surgical Airway Reduced by a Comprehensive Difficult Airway Program," Anesthesia \& Analgesia, Vol. 109, No. 6, 2009, pp. 1860-1869. http://dx.doi.org/10.1213/ane.0b013e3181b2531a

[13] M. L. Sivilotti, D. W. Messenger, J. van Vlymen, P. E. Dungey and H. E. Murray, "A Comparative Evaluation of Capnometry Versus Pulse Oximetry During Procedural Sedation and Analgesia on Room Air,” Canadian Journal of Emergency Medicine, Vol. 12, No. 5, 2010, pp. 397404.

[14] S. L. Chritton, J. Weinkauf, C. D. McClain, J. E. O'Flaherty, P. Livingston, F. M. Evans, S. Kessler, E. P. Nelson and M. E. Durieux, "Teaching Anesthesiology in Rwanda: A Multi-pronged Approach,” American Society of Anesthesiologists Newsletter, Vol. 77, 2013, pp. 52-55.

[15] M. J. Rice, A. Gwertzman, T. Finley and T. E. Morey, "Anesthetic Practice in Haiti after the 2010 Earthquake," Anesthesia \& Analgesia, Vol. 111, No. 6, 2010, pp. 11451149. http://dx.doi.org/10.1213/ANE.0b013e3181fa3241

[16] M. McCunn, M. A. Ashburn, T. F. Floyd, C. W. Schwab, P. Harrington, C. W. Hanson, et al., "An Organized, Comprehensive, and Security-Enabled Strategic Response to The Haiti Earthquake: A Description of Pre-Deployment Readiness Preparation and Preliminary Experience From an Academic Anesthesia Department with No Preexisting International Disasters Response Program," Anesthesia \& Analgesia, Vol. 111, No. 6, 2010, pp. 14381444.http://dx.doi.org/10.1213/ANE.0b013e3181f42fd3

[17] A. L. Kushner, M. N. Cherian, L. Noel, D. A. Spiegal, S. Groth and C. Etienne, “Addressing The Millennium Development Goals from a Surgical Perspective: Essential Surgery and Anesthesia in 8 Low and Middle Income Countries," Archives of Surgery, Vol. 145, No. 2, 2010, pp. 154-160. http://dx.doi.org/10.1001/archsurg.2009.263

[18] C. Doherty and H. Holtby, "Pediatric Cardiac Anesthesia in the Developing World," Pediatric Anesthesia, Vol. 21, No. 5, 2011, pp. 609-614. http://dx.doi.org/10.1111/j.1460-9592.2010.03492.x

[19] S. M. Green, K. J. Clem and S. G. Rothrock, "Ketamine Safety Profile in the Developing World: Survey of Practitioners,” Academic Emergency Medicine, Vol. 3, No. 6, 1996, pp. 598-604.

http://dx.doi.org/10.1111/j.1553-2712.1996.tb03470.x

[20] I. A. Walker, A. F. Merry, I. H. Wilson, G. A. McHugh, E. O’Sullivan, G. M. Thoms, et al., "Global Oximetery: An International Anesthesia Quality Improvement Project,” Anesthesia, Vol. 64, No. 10, 2009, pp. 1051-1060. http://dx.doi.org/10.1111/j.1365-2044.2009.06067.x

[21] S. Jochberger, O. Bataar, N.Mendsaikhan, W. Grander, G. Tsenddori, G. Lundeg, et al., "Anesthesia Care in A Medium-Developed Country: A Nationwide Survery of Mongolia,” Journal of Clinical Anesthesia, Vol. 22, No. 6, 2010, pp. 443-449.

http://dx.doi.org/10.1016/j.jclinane.2009.12.005

[22] O. A. Ogunbiyi and C. N. Mato, "Anesthetic Accidents: An Experience in Sub-Saharan Africa,” Southern African Journal of Anaesthesia \& Analgesia, Vol. 12, No. 3, 2006, pp. 111-113.

[23] J. B. Cooper, R. S. Newbower and R. J. Kitz, “An Analysis of Major Errors and Equipment Failures in Anaesthesia Management: Considerations for Prevention and Detection,” Anesthesiology, Vol. 60, No. 1, 1984, pp. 34-42. http://dx.doi.org/10.1097/00000542-198401000-00008

[24] A. Gawande, "The Check List Manifesto: How to Get Things Right,” Metropolitan Books, New York, 2010. 
[25] J. E. Ziewacz, A. F. Arriaga, A. M. Bader, W. R. Berry, L. Edmondson, J. M. Wong, et al., "Crisis Checklists for the Operating Room: Development and Pilot Testing," Journal of American College of Surgeons, Vol. 213, No. 2, 2011, pp. 212-217. http://dx.doi.org/10.1016/j.jamcollsurg.2011.04.031

[26] J. M. Ehrenfeld, L. M. Funk, J. VanSchalkwyk, F. Merry, W. S. Sandbergand A. A. Gawande, "The Incidence of Hypoxemia during Surgery: Evidence from Two Institutions," Canadian Journal of Anesthesia, Vol. 57, No. 10, 2012, pp. 888-897. http://dx.doi.org/10.1007/s12630-010-9366-5

[27] C. L. Parks and M. E. Schroeder, "Military Anesthesia
Trainees in WWII at the University of Wisconsin: Their Training, Careers, and Contributions," Anesthesiology, Vol. 118, No. 5, 2013, pp. 1019-1027.

http://dx.doi.org/10.1097/ALN.0b013e318286d0e2

[28] A. Schubert, G. Eckhout and K. Tremper, "An Updated View of the National Anesthesia Personnel Shortfall," Anesthesia \& Analgesia, Vol. 96, 2003, pp. 207-214.

[29] D. Marchbein, "Humanitarian Surgery: A Call to Action for Anesthesiologists,” Anesthesiology, Vol. 119, No. 5, 2013, pp.1001-1002.

http://dx.doi.org/10.1097/ALN.0b013e3182a950fb 